\title{
Criptosporidiose em caprinos: A doença e seus impactos na produção
}

\author{
[Cryptosporidiosis in goats: The disease and its impacts on production]
}

\section{"Revisão/Review"}

\author{
Marcelo Richelly Alves de Oliveira ${ }^{1 *}$, Carlos Syllas Monteiro Luz ${ }^{1}$, \\ José Elivalto Guimarães Campelo², Karina Rodrigues dos Santos ${ }^{3}$, \\ Severino Cavalcante de Sousa Júnior ${ }^{3}$
}

\begin{abstract}
1Programa de Pós-Graduação em Ciência Animal, Universidade Federal do Piauí, Teresina-PI, Brasil. ${ }^{2}$ Departamento de Zootecnia, Universidade Federal do Piauí, Teresina-PI, Brasil. ${ }^{3}$ Departamento de Medicina, Universidade Federal do Piauí, Parnaíba-PI, Brasil.

*Autor para correspondência/Corresponding author: E-mail: marcelo-zootec@ hotmail.com
\end{abstract}

\begin{abstract}
Resumo
A Criptosporidiose é uma zoonose parasitária de distribuição cosmopolita. O Cryptosporidium spp., agente etiológico da doença, tem localização intracelular, completa seu ciclo biológico na superfície de células epiteliais dos tratos respiratório e gastrointestinal e são responsáveis pela síndrome da "diarreia aquosa", acarretando em desidratação, perda de peso, retardo no crescimento e morte dos indivíduos infectados. A Criptosporidiose ocorre com maior gravidade em animais neonatos e/ou imunocomprometidos, causando grandes prejuízos econômicos. Os protozoários do gênero Cryptosporidium spp., apresentam ciclo de vida monoxeno, ou seja, o realizam em um único hospedeiro. Em caprinos jovens, menores de 30 dias de vida, o sinal clínico habitualmente observado é o de diarreia severa, que pode durar de 5 a 20 dias comprometendo o crescimento destes indivíduos e podendo levá-los à óbito. O diagnóstico da Criptosporidiose em caprinos pode ser realizado com o uso de técnicas de coloração em esfregaço fecal e Centrífugo-flutuação, de fácil execução e baixo custo, ou ainda por técnicas mais modernas e precisas como a PCR. Pelo conteúdo exposto nesta revisão, é possível concluir que apesar da Criptosporidiose ser uma enfermidade crônica e ainda não ter tratamento efetivo para o combate do seu agente etiológico, tem na prevenção a principal forma de evitar a disseminação da doença no rebanho caprino.
\end{abstract}

Palavras-chave: Cryptosporidium spp.; diarreia; protozoários; zoonose.

\begin{abstract}
Cryptosporidiosis is a cosmopolitan parasitic zoonotic disease caused by the etiological agent Cryptosporidium spp., which has an intracellular location. This agent completes its biological cycle in the surface of epithelial cells of the respiratory and gastrointestinal tracts, being responsible for the syndrome characterized by watery diarrhea, dehydration, weight loss, slow growth, and death of infected individuals. Cryptosporidiosis occurs with greater severity in neonatal and immunocompromised animals, causing great economic losses. Cryptosporidium spp. protozoan have a monoxene life cycle, i.e., they have only one host. In goats under 30 days of age, the usual clinical sign consists in severe diarrheas, which can last 5 to 20 days, affecting the growth of these individuals, which may lead to death. There are several techniques for diagnosis of cryptosporidiosis in goats, such as the use of stained fecal smear and centrifugal flotation, which are easy to perform and inexpensive. In addition, more modern and accurate techniques such as PCR are available for the purpose mentioned above. From the reviewed literature, it is possible to conclude that, although cryptosporidiosis is a chronic disease and still has no effective treatment to combat its etiological agent, the prevention of this disease is the main way to prevent its spread in goat herds.
\end{abstract}

Keywords: Cryptosporidium spp.; diarrhea; goats; infection; protozoan; zoonosis. 


\section{Introdução}

Em várias regiões do mundo as endoparasitoses, principalmente as localizadas no trato gastrointestinal, são um dos principais entraves à produção de caprinos (Teixeira et al., 2015). Além das helmintoses, os protozoários também são considerados como uma importante classe de parasitos que podem acometer o trato gastrointestinal de pequenos ruminantes dentre outras espécies, como os protozoários do gênero Cryptosporidium spp., que causam uma doença denominada Criptosporidiose.

A criptosporidiose constitui-se como uma zoonose parasitária de distribuição cosmopolita (Ó Santos et al., 2016; Rodrigues et al., 2016) sendo seus agentes etiológicos considerados parasitos oportunistas, tem localização intracelular obrigatória e completam seu ciclo biológico na superfície de células epiteliais dos tratos respiratório e gastrointestinal. São responsáveis pela síndrome de "diarreia aquosa", causando dores abdominais, desidratação, perda de peso, retardo no crescimento, acometendo com maior gravidade indivíduos neonatos e/ou animais imunocomprometidos, causando grandes prejuízos econômicos morte (Galvão et al., 2012; Bresciani et al., 2013; Lima et al., 2013).

Os protozoários do gênero Cryptosporidium spp. não são espécie-específicos, portanto, pode haver transmissão de diferentes espécies de animais (domésticos, silvestres, aquáticos e répteis) para os seres humanos. O oocisto, seu estágio infectante, é liberado com as fezes e permanece viável por vários meses e a principal via de transmissão é a fecal-oral, estando associada à ingestão de água, de alimentos e contato com animais e/ou humanos infectados (Huber et al., 2007; Smith et al., 2007; Rossi et al., 2014).

Em caprinos, parasitos deste gênero podem acometer indivíduos em diferentes idades, sexo e padrão racial, sendo que a infecção é mais comum em animais neonatos e/ou com até cinco meses de vida (Noordeen et al., 2012). Como este protozoário parasita principalmente $\mathrm{o}$ trato gastrointestinal dos animais, o mesmo, causa danos ao epitélio intestinal, prejudicando a absorção dos nutrientes, o que leva a perda de peso e retardo no crescimento dos indivíduos acometidos (Soltane et al., 2007). Assim, o Cryptosporidium spp. é considerado um dos principais agentes patogênicos que causam diarreia em caprinos, sendo que sua incidência em rebanhos desta espécie, promove redução significativa nos índices de produtividade, como queda no ganho de peso e na produção de leite (Delafosse et al., 2006; Noordeen et al., 2012).

É relevante a realização de pesquisas sobre a presença de protozoários do gênero Cryptosporidium spp. em rebanhos caprinos, devido aos prejuízos financeiros causados pela infecção e aos riscos à saúde pública que esta enfermidade pode oferecer, uma vez que os caprinos podem servir como reservatório deste parasita que infectam o homem, como a C. parvum (Bresciani et al., 2013).

Diante do exposto, objetivou-se com esta revisão discorrer sobre a infecção de caprinos por protozoários do gênero Cryptosporidium spp. no comprometimento da sanidade e por consequência, dos índices produtivos do rebanho acometido.

\section{Desenvolvimento \\ Histórico da doença}

A infecção por protozoários do gênero Cryptosporidium spp. foi citada pela primeira vez nos Estados Unidos por Tyzzer (1907), quando identificou em camundongos a espécie $C$. muris. Anos depois, em 1912, o autor identificou outra espécie de Cryptosporidium, também em camundongos, a C. parvum.

Segundo Carneiro et al. (1995), estes protozoários são comumente encontrados nas vilosidades do intestino e medem cerca de 3 a 4 micrômetros de diâmetro. $\mathrm{O}$ gênero Cryptosporidium spp. é composto por 20 espécies e mais de 40 genótipos (Tzanidakis et al., 2014). Como o agente etiológico da Criptosporidiose não é espécie-específico, este pode infectar várias espécies de animais domésticos e/ou silvestres.

Diversos estudos identificaram a infecção por protozoários do gênero Cryptosporidium spp. em: caprinos (Capra hircus), bovinos (Bos taurus/indicus) e ovinos (Ovis aries) (Ayana e Alemu, 2015); frangos de corte (Gallus gallus) (Jacobsen et al., 2006); suínos (Sus domesticus) (Fiuza et al., 2009); diferentes espécies de roedores (Akodon montensis, Thaptomys nigita, Sciurus aestuans) (Lallo et al., 2009); de serpentes (Boa constrictor amaralis, Boa jararaca, Caudisona durissa) (Custódio Ruggiero et al., 2011); gato doméstico (Felis catus) (Pereira et al., 2012); puma (Puma concolor) (Fanfa et al., 2011); peixe-boi marinho (Trichechus manatus) (Borges et al., 2009) dentre outras espécies.

Por se tratar de uma zoonose, a Criptosporidiose também pode acometer o homem. 
O primeiro registro desta infecção em humanos foi feito por Nime et al. (1976) em um indivíduo com idade de 3 anos e que apresentava alguns sintomas, como vômitos, dores no abdômen e diarreia. Ainda em 1976, Meisel et al., identificaram o protozoário em pacientes imunossuprimidos, constatando assim, algumas pré-disposições para o risco da infecção em humanos, como a idade e o estado imunológico do indivíduo candidato à infecção pelo parasito.

A criptosporidiose em humanos possui diferentes agentes etiológicos, mas as espécies de Cryptosporidium mais comumente encontradas são a $C$. hominis e $C$. parvum, que apresentam dois ciclos de transmissão distintos: o ciclo antroponótico, em que a espécie envolvida seria a C. hominis e o ciclo zoonótico, que envolveria duas espécies distintas, principalmente a espécie de Cryptosporidium parvum (Dabas et al., 2017).

\section{Ciclo de vida do parasito e via de transmissão da doença}

Os protozoários do gênero Cryptosporidium spp., pertencem ao Filo Apicomplexa, Classe Sporozoa, Subclasse Coccidia, Ordem Eucoccidiida, Subordem Eimeriina e Família Cryptosporidiidae (De Carvalho, 2009; Brito et al., 2014), apresentam ciclo de vida monoxeno, ou seja, o realizam em um único hospedeiro e que pode ser descrito em quatro diferentes fases (Martins-Vieira et al., 2009).

Fase 1 - Desencistamento: esse é o início da fase endógena, quando os esporozoítos, em número de quatro, são liberados do oocisto e penetra no trato digestivo organismo do hospedeiro (via oral ou nasal), fixando-se em seguida nas microvilosidades do intestino (Ortolani, 2000; Cabral et al., 2001; Martins-Vieira et al., 2009).

Fase 2 - Merogonia ou Esquizogonia: é a fase assexuada do ciclo do Cryptosporidium spp., ocorrendo a evolução dos esporozoítos para esquizontes e merócitos, tendo em seu interior quatro ou oito merozoítos. Com o rompimento do merócito, ocorre a liberação dos merozoítos para parasitarem novas células. Após a ocorrência de duas gerações nesta fase, inicia-se a reprodução sexuada (gametogonia) (Ortolani, 2000; Cabral et al., 2001; Martins-Vieira et al., 2009).

Fase 3 - Gametogonia (fase sexuada): Nesta etapa do ciclo, os merozoítos de segunda geração se distinguem em macro e microgametócito depois de sua entrada no enterócito, que após sua maturação, ocorre a liberação de microgametas.
Com a divisão do núcleo no microgametócito, ocorre formação dos núcleos do macro e do microgameta, que ao se fundirem darão origem ao zigoto, que se diferencia em oocisto (Ortolani, 2000; Cabral et al., 2001; Martins-Vieira et al., 2009).

Fase 4 - Esporulação: Nesta fase, cada oocisto apresenta quatro esporozoítos em seu interior. Estes oocistos podem ser formados de duas formas: oocisto de parede fina e oocisto de parede grossa ou espessa. O de parede fina, representa em torno de $20 \%$ do total de oocistos presentes e se rompem no interior do mesmo indivíduo, causando o que se pode chamar de autoinfecção interna. Ao passo que, os $80 \%$ que restam são de oocistos de parede espessa, sendo os deste tipo liberados no meio ambiente e, por apresentarem essa característica, possuem maior resistência às intempéries ambientais (Ortolani, 2000; Cabral et al., 2001; Martins-Vieira et al., 2009).

A mais relevante forma do Cryptosporidium spp. dentro de seu ciclo de desenvolvimento é a de oocisto esporulado, ou seja, quando estes são eliminados nas fezes humanas e/ou dos animais infectados (Dabas et al., 2017). Suas formas de transmissão são de animal para animal, dos animais para o homem e de pessoa para pessoa, tendo por via principal a fecal-oral, dada comumente pelo consumo de água e alimentos contaminados (Bresciani et al., 2013).

\section{Espécies de Cryptosporidium spp. que acometem caprinos}

O primeiro relato de Criptosporidiose em caprinos ocorreu na Austrália, pela observação da enfermidade em um animal jovem com idade de duas semanas, que apresentou um quadro de diarreia aguda antes de vir a óbito (Mason et al., 1981).

O número de espécies que podem infectar caprinos é variado, pois estudos realizados por Geurden et al. (2008) comprovaram com o auxílio de PCR e utilizando um gene da glicoproteína $60 \mathrm{kDa}$ em caprinos jovens, a presença dos subgenótipos do gênero Cryptosporidium IIa15G2R1 e o IIdA22G1, que apresentam maior e menor potencial zoonótico, respectivamente. Por este motivo, a espécie Cryptosporidium parvum subgenótipo IIa15G2R1, apresenta grande relevância para saúde pública.

$\mathrm{Mi}$ et al. (2014) identificaram em investigação realizada em caprinos de diferentes 
idades, as espécies de $C$. xiao, $C$. parvum e $C$. ubiqitum, sendo que a maior porcentagem de animais positivos foi observada em caprinos no pós-desmame. A espécie de $C$. xiaoi, também foi encontrada em estudo desenvolvido por Parsons et al. (2015) e Kaupke et al. (2017), que ao pesquisarem a infecção por este gênero de protozoários em caprinos, identificando pela primeira nessa espécie, o subtipo de Cryptosporidium IIdA23G1.

Paul et al. (2014), citam a espécie $C$. hominis, com potencial para infectar caprinos, relatando ainda que, embora comumente relacionada à espécie bovina, a espécie de $C$. bovis é também encontrada em caprinos, haja vista que protozoários deste gênero não são espécieespecíficos, sendo possível a infecção entre espécies de parasito e de animais hospedeiros distintos, principalmente quando estes compartilham o mesmo ambiente.

Diante dos estudos realizados até 0 momento, fica evidente que os caprinos têm um importante papel na transmissão de protozoários do gênero Cryptosporidium spp. para os seres humanos, e o fato desses animais serem possíveis reservatórios de C. parvum, espécie que possui um importante potencial zoonótico cientificamente comprovado, torna ainda mais relevante a realização de estudos mais aprofundados da doença nessa espécie doméstica (Quílez et al., 2008; Bresciani et al., 2013; Rieux et al., 2013).

\section{Patogenia e sinais clínicos da doença na espécie caprina}

A patogenia da infecção por Cryptosporidium spp. não está bem definida (Paul et al., 2014), sabendo-se apenas que o principal sinal clínico ligado à doença é a diarreia causada pela digestão e absorção deficientes, pela redução da ação de enzimas digestivas e pelo comprometimento das microvilosidades e do epitélio intestinal dos indivíduos acometidos (Bresciani et al., 2013). Observa-se que os danos causados pelo parasito à mucosa intestinal estão ligados ao aumento da permeabilidade paracelular no órgão, e ao comprometimento da função de barreira da mucosa que reveste o intestino (Klein et al., 2008; Paul et al., 2014).

Em caprinos, a manifestação da doença ocorre de modo diferente, dependendo da idade, pois em animais adultos não é comum o aparecimento dos sinais clínicos supracitados, tendo importância epidemiológica por serem fontes de infecção para animais jovens da espécie (Brito et al., 2014). Em animais menores de 30 dias de vida, o sinal clínico habitualmente observado é o de diarreia severa que pode durar de 5 a 20 dias, podendo comprometer o crescimento destes indivíduos caso não venham à óbito (Brito et al., 2014; Ayana e Alemu, 2015).

\section{Formas de diagnóstico da Criptosporidiose}

Existem algumas formas de detecção da presença de protozoários do gênero Criptosporidium spp. em amostras fecais de diversas espécies de animais, inclusive a caprina, que vão desde técnicas de esfregaço fecal até análises mais modernas e precisas, principalmente no que diz respeito à identificação da espécie, como os métodos de biologia molecular.

Esfregaço fecal: A detecção da presença de oocistos de Cryptosporidium spp. por coloração em esfregaço fecal é de fácil acesso, baixo custo de execução e resultado rápido. Pode ser realizada comumente com a técnica de coloração com fucsina carbólica de Ziehl-Neelsen (Henricksen e Pohlenz, 1981) e suas variações, como Kinyoun modificado a frio, Ziehl-Neelsen modificado a quente, Ziehl-Neelsen-Dimetilsulfóxido e Safranina modificada a quente, todas elas têm como princípio corar os oocistos do protozoário com coloração vermelho-alaranjada brilhante, quando estão presentes nas amostras de fezes de animais parasitados (Huber et al., 2004), como fizeram Jafari et al. (2012) e Giadinis et al. (2015), ao detectarem a presença de oocistos de Cryptosporidium spp. em caprinos.

Centrífugo-Flutuação: A técnica de centrífugo-flutuação consiste no uso de solução de açúcar para que os oocistos sejam separados da massa fecal e identificados. Os oocistos contidos em amostras de fezes frescas podem ser observados ligeiramente róseos na microscopia de campo claro, e claros e refringentes quando vistos em contraste de fase (Huber et al., 2004). Essa técnica foi utilizada em estudo desenvolvido por Ayana e Alemu (2015), ao analisarem amostras fecais de cabritos na Etiópia, bem como utilizada por Pam et al. (2013) pesquisando parasitos em caprinos no Irã.

ELISA: O teste ELISA, do inglês "EnzymeLinked Immunosorbent Assay" (ensaio de imunoabsorção enzimática), tem como base as reações antígeno-anticorpo, que são detectáveis através de reações enzimáticas e são mais sensíveis e específicos que as técnicas parasitológicas 
tradicionais (Brito et al., 2014). Foi utilizando um teste comercial de ELISA em pesquisa desenvolvida por Akinkuoto et al. (2015), que puderam detectar coproantígenos da espécie Cryptosporidium parvum em amostras fecais de caprinos em diferentes idades, com e sem a presença de sinal clínico de diarreia.

PCR: O uso de técnicas moleculares, como a reação em cadeia de polimerase (PCR), tornou possível a análise genômica do gênero Cryptosporidium spp. (Wang et al., 2012; Brito et al., 2014; Feng e Xiao, 2017). As principais moléculas utilizadas para duplicação do DNA do protozoário e que possuem a capacidade de tornar viável a classificação das diferentes espécies e de seus genótipos, são a subunidade conhecida por $18 \mathrm{~S}$ do gene RNA, a proteína de parede do oocisto de Cryptosporidium spp., a proteína de choque térmico $70 \mathrm{Kda}$, além dos genes da $\beta$ tubulina e actina. A análise de genotipagem de Cryptosporidium spp. é baseado na amplificação dos fragmentos da subunidade rRNA (Brito et al., 2014; Feng e Xiao, 2017).

\section{Prejuízos causados à saúde e à produção de caprinos}

As perdas econômicas causadas pela Criptosporidiose podem afetar o rebanho caprino de modo direto e/o indireto (Brito et al., 2014), uma vez que os prejuízos à sanidade bem como aos índices produtivos no rebanho caprino (Paul et al., 2014; Rossi et al., 2014) estão intimamente ligados aos danos causados ao trato gastrointestinal desses animais, principalmente ao intestino, órgão onde ocorre a maior parte da absorção dos nutrientes, que por sua vez, se converterão em proteína como leite e carne.

Como um dos principais sinais clínicos associados à Criptospodiose, a diarreia pode levar a lesões entéricas que dificultam a absorção dos nutrientes e comprometem a conversão alimentar, acarretando na perda de peso principalmente em animais neonatos (Quilez et al., 2008; Bresciani et al., 2013; Tzanidakis et al., 2014).

Em fêmeas adultas, comumente assintomáticas, a infecção pode causar redução na produção de leite, além de eventuais custos com tratamentos quando sinais clínicos são apresentados pelos animais infectados (Mahfouz et al., 2014). Em cabritos, tal problema pode levar ao retardo no crescimento, anorexia, e em casos mais graves, a óbito (Akinkuotu et al., 2015).
Em cabritos naturalmente infectados por Cryptosporidium parvum, foi relatada uma diferença em ganho de peso de até $2 \mathrm{~kg}$, num período de 1 mês, quando comparado à animais sadios (De Graaf et al., 1999, citado por Pam et al., 2013). Segundo Brito et al. (2014), as citadas perdas observadas na produção de caprinos estão ligadas principalmente, a falhas no manejo sanitário e em animais criados sob sistemas intensivos de criação.

\section{Prevenção e tratamento da Criptosporidiose em caprinos}

Até o momento não existem medicamentos disponíveis, capazes de controlar de forma efetiva a infecção por protozoários do gênero Cryptosporidium spp. na espécie caprina. O que pode ser feito para impedir sua transmissão e controlar a disseminação no rebanho, é adotar medidas sanitárias e profiláticas (Féres et al., 2009), como, por exemplo, a retirada das fezes e camas da área destinadas aos cabritos neonatos e lactentes, (devido a relação direta das fezes de animais infectados como principal via de transmissão da doença), que devem ser descartadas em locais apropriados, como biodigestores e/ou esterqueiras, realizar diariamente a limpeza de comedouros, bebedouros e saleiros, de preferência que estes equipamentos sejam instalados do lado de fora dos abrigos, no intuito de evitar que os animais excretem e contaminem a água e os alimentos, isolar animais com suspeita de infecção por Cryptosporidium spp. ou comprovadamente infectados para realização do tratamento adequado (Féres et al., 2009).

Em casos de confirmação da Cripstosporidiose, recomenda-se descartar os animais do rebanho para evitar a propagação da doença, principalmente pelo fato de se tratar de uma infecção crônica (Rossi et al., 2014; Brito et al., 2014). Sendo importante ressaltar que ainda não foi desenvolvida nenhuma vacina eficaz ou medicamentos específicos para o combate da Criptosporidiose. No entanto, vários fármacos foram avaliados por suas ações criptosporicidas, dentre eles, o Decoquinato (Ferre et al., 2005), Nitazoxanida (Viel et al., 2007) e Lactato de Halofuginona (Giadinis et al., 2008).

\section{Considerações Finais}

De acordo com o exposto nesta revisão, é possível concluir que apesar da Criptosporidiose ser uma enfermidade crônica em caprinos adultos, 
mas, aguda em jovens, e ainda não ter tratamento efetivo para o combate do seu agente etiológico, tem na prevenção a principal forma de evitar a disseminação da doença na criação de caprinos.

Dentre as medidas profiláticas adotadas para prevenir a proliferação da criptosporidiose no rebanho caprino, podem ser destacadas: a higiene periódica das instalações como comedouros e bebedouros, separar animais segundo a faixa etária, evitando o contato direto de animais jovens com animais adultos, evitar o contato de outras espécies animais junto ao rebanho como aves, cães, gatos, etc., realizar testes diagnósticos em animais com suspeita da doença, isolar e realizar a eutanásia de animais comprovadamente infectados, dentre outras.

\section{Referências}

Akinkuotu, O.A.; Fagbemi, B.O.; Egbetade, A.O.; Jacobs, E.B.; Adeyanju, J. Prevalence of Cryptosporidium coproantigens in goats in Ogun, Southwest Nigeria. Journal of Veterinary Advances, 5(10): 1122-1126, 2015.

Ayana, D.; Alemu, B. Cryptosporidiosis in calves, lambs and goat kids in Bishoftu, Oromia Regional State, Ethiopia. African Journal of Basic \& Applied Sciences, 7(5): 233-239, 2015.

Borges, J.C.G.; Alves, L.C.; Vergara-Parente, J.E.; Faustino, M.A.D.G.; Machado, E.D.C.L. Ocorrência de infecção Cryptosporidium spp. em peixe-boi marinho (Trichechus manatus). Revista Brasileira de Parasitologia Veterinária, 18(1): 60-61, 2009.

Bresciani, K.D.S.; de Aquino, M.C.C.; Zucatto, A.S.; Inácio, S.V.; da Silveira Neto, L.;Coelho, N.M.D.; Meireles, M.V. Criptosporidiose em animais domésticos: aspectos epidemiológicos. Semina: Ciências Agrárias, 34(5): 2387-2402, 2013.

Brito, R.L.L.; Inácio, S.V.; Oliveira, D.D.S.; de Sousa, M.M.; Meireles, M.V.; Lobo, R.N.B.; Bresciani, K.D.S. Ocorrência da infecção por Cryptosporidium spp. em cabritos (Capra hircus). Embrapa Caprinos e Ovinos-Artigo em periódico indexado (ALICE), 2014.

Cabral, D.D.; Barbosa, F.C.; Strasser, C.; Barsotti, S.R.H. Exame de fezes de mamíferos silvestres para verificação de parasitismo por Cryptosporidium sp. Bioscience Journal, 17(1): 77-83, 2001.

Carneiro, J.R.; Rodrigues, N.; Lima, J.D.; Evangelista, P.L. Prevalência da Criptosporidiose em crianças procedentes da região metropolitana de Goiânia - GO. Revista de Patologia Tropical, 24(2): 243-253, 1995.
Ruggiero, P.C.; Zacariotti, R.L.; Bondan, E.F.; Lallo, M.A. Prevalência de Cryptosporidium serpentis em serpentes de cativeiro. Ciência Rural, 41(11): 1975-1978, 2011.

Dabas, A.; Shah, D.; Bhatnagar, S.; Lodh, R. Epidemiology of Cryptosporidium in pediatric diarrheal illnesses. Indian Pediatrics, 54(4): 299-309, 2017.

De Carvalho, T.T.R. Estado atual do conhecimento de Cryptosporidium e Giardia. Revista de Patologia Tropical, 38(1): 01-16, 2009.

De Graaf, D.C.; Vanopdenbosch, E.; Ortega-Mora, L.M.; Abbassi, H.; Peeters, J.E. A review of the importance of cryptosporidiosis in farm animals. International Journal for parasitology, 29(8): 1269-1287, 1999.

Delafosse, A.; Castro-Hermida, J.A.; Baudry, C.; Ares-Mazas, E.; Chartier, C. Herd-level risk factors for Cryptosporidium infection in dairygoat kids in western France. Preventive Veterinary Medicine, 77(1-2): 109-121, 2006.

Fanfa, V.; Farret, M.; Da Silva, A.S.; Monteiro, S. Endoparasitoses em puma (Puma concolor) na região Sul do Brasil. Acta Veterinaria Brasilica, 5(1): 100-102, 2011.

Feng, Y.; Xiao, L. Molecular epidemiology of cryptosporidiosis in China. Frontiers in Microbiology, 8(1701):1-11, 2017.

Féres, F.C.; Lombardi, A.L.; Carvalho, M.P.P.; Mendes, L.C.N.; Peiró, J.R.; Cadioli, F.A.; Meireles, M.V.; Perri, S.H.V.; Feitosa, F.L.F. Ocorrência e caracterização molecular de Cryptosporidium em cordeiros. Arquivo Brasileiro de Medicina Veterinária e Zootecnia, 61(4): 1002-1005, 2009.

Ferre, I.; Benito-Peña, A.; García, U.; Osoro, K.; Ortega-Mora, L.M. Effect of different decoquinate treatments on cryptosporidiosis in naturally infected cashmere goat kids. Veterinary Record, 157(9): 261, 2005.

Fiuza, V.R.D.S.; Cosendey, R.I.J.; Pimentel, F.F.; Oliveira, F.C.R.D. "Cryptosporidium" spp. em suínos de granjas familiares e tecnificadas das regiões norte e noroeste do Estado do Rio de Janeiro. Revista Brasileira de Saúde e Produção Animal, 10(2): 356-365, 2009.

Galvão, A.L.B.; Ortiz, E.G.; Ferreira, G.S.; de Vasconcellos, A.L.; Vieira, M.C.; Bresciani, K. D.S. Importância da criptosporidiose como zoonose. Archives of Veterinary Science, 17(2): 18-28, 2012.

Geurden, T.; Thomas, P.; Casaert, S.; Vercruysse, J.; Claerebout, E. Prevalence and molecular characterization of Cryptosporidium and Giardia in lambs and goat kids in Belgium. 
Veterinary Parasitology, Amsterdam, 155(12): 142-145, 2008.

Giadinis, N.D.; Papadopoulos, E.; Lafi, S.Q.; Panousis, N.K.; Papazahariadou, M.; Karatzias, $\mathrm{H}$. Efficacy of halofuginone lactate for the treatment and prevention of cryptosporidiosis in goat kids: an extensive field trial. Small Ruminant Research, 76(3): 195-200, 2008.

Giadinis, N.D.; Papadopoulos, E.; Lafi, S.Q.; Papanikolopoulou, V.; Karanikola, S.; Diakou, A.; Karatzias, H. Epidemiological observations on cryptosporidiosis in diarrheic goat kids in Greece. Veterinary Medicine International, 2015:1-4, 2015.

Henriksen, S.; Pohlenz, I.J. Staining of cryptosporidia by a modified Zielh-Neelsen technique. Acta Veterinaria Scandinavica, 22(3-4): 594-596, 1981.

Huber, F.; Bomfim, T.C.; Gomes, R.S. Comparação da eficiência da coloração pelo método da safranina a quente e da técnica de centrífugoflutuação na detecção de oocistos de Cryptosporidium em amostras fecais de animais domésticos. Revista Brasileira de Parasitologia Veterinária, 13(2): 81-84, 2004.

Huber, F.da Silva, S.; Bomfim, T.C.B.; Teixeira, K.R.S.; Bello, A.R. Genotypic characterization and phylogenetic analysis of Cryptosporidium sp. from domestic animals in Brazil. Veterinary Parasitology, 150(1): 65-74, 2007.

Jacobsen, G.; Barcelos, A.D.S.; Flôres, M.L.; Segabinazi, S.D.; Lagaggio, V.R.A. Cryptosporidium sp. in intestines, bursa of Fabricius and poultry trachea (Gallus gallus sp). Ciência Rural, 36(2): 682-684, 2006.

Jafari, R.; Maghsood, A.H.; Fallah, M. Prevalence of Cryptosporidium infection among livestock and humans in contact with livestock in Hamadan district, Iran, 2012. Journal of Research in Health Sciences, 13(1): 86-89, 2012.

Kaupke, A.; Michalski, M.M.; Rzeżutka, A. Diversity of Cryptosporidium species occurring in sheep and goat breeds reared in Poland. Parasitology Research, 116(3): 871-879, 2017.

Klein P.; Kleinova T.; Volek, Z.; Simunek, J. Effect of Cryptosporidium parvum infection on the absorptive capacity and paracellular permeability of the small intestine in neonatal calves. Veterinary Parasitology, 152(1-2): 5359, 2008.

Lallo, M.A.; Pereira, A.; Araújo, R.; Favorito, S.E.; Bertolla, P.; Bondan, E.F. Ocorrência de Giardia, Cryptosporidium e microsporídios em animais silvestres em área de desmatamento no
Estado de São Paulo, Brasil. Ciência Rural, 39(5): 1465-1470, 2009.

Lima, R.C.A.; Aquino, M.C.C.; Inácio, S.V.; Viol, M.A.; Zucatto, A.S.; Silveira Neto, L.D.; Costa, A.J. Caracterização molecular de Cryptosporidium spp. em bezerros (Bos taurus e Bos indicus) no município de Formiga, Minas Gerais-Brasil. Semina: Ciências Agrárias, 34(6): 3747-3754, 2013.

Mahfouz, M.E.; Mira, N.; Amer, S. Prevalence and genotyping of Cryptosporidium spp. in farm animals in Egypt. Journal of Veterinary Medical Science, 76(12): 1569-1575, 2014.

Martins-Vieira, M.B.C.; Brito, L.A.L.; Heller, L. Oocistos de Cryptosporidium parvum em fezes de bezerro infectado experimentalmente. Arquivo Brasileiro de Medicina Veterinária e Zootecnia, 61(6): 1454-1458, 2009.

Mason, R.W.; Hartley, W.J.; Tilt, L. Intestinal cryptosporidiosis in a kid goat. The Journal of the Australian Veterinary Association, 57(8): 386-388, 1981.

Meisel, J.L.; Perera, D.R.; Meligro, C.; Rubin, C.E. Overwhelming watery diarrhea associated with Cryptosporidium in an immunosupressed patient. Gastroenterology, 70(6): 1156-1160, 1976.

Mi, R.; Wang, X.; Huang, Y.; Zhou, P.; Liu, Y.; Chen, Y.; Chen, Z. Prevalence and molecular characterization of Cryptosporidium in goats across four provincial level areas in China. PloS One, 9(10): 1-7, 2014.

Nime, F.A.; Burek, J.D.; Page, D.L.; Holsher, M.A.; Yardley, J.H. Acute enterocolitis in a human being infected with protozoan Cryptosporidium. Gastroenterology, 70(4): 592-598, 1976.

Noordeen, F.; Rajapakse, R.P.V.J.; Horadagoda, N.U.; Abdulcareem, M.F. Cryptosporidium, an important enteric pathogen in goats-A review. Small Ruminant Research, 106(2-3): 77-82, 2012.

Ó Santos, R.; Oliveira, M.R.A.; Luz, C.S.M.; Silva Abreu, B.; Sousa Júnior, S.C.; Santos, K.R. Occurence of protozoan from the genus Cryptosporidium spp. in cattle raised in properties of the rural zone in the county of Bom Jesus, Piauí. Acta Veterinaria Brasilica, 10(4): 346-351, 2016.

Ortolani, E. L. Standardization of the modified ZiehlNeelsen technique to stain oocysts of Cryptosporidium sp. Revista Brasileira de Parasitologia Veterinária, 9(1): 29-31, 2000.

Pam, V.A.; Dakul, D.A.; Karshima, N.S.; Bata, S.I.; Ogbu, K.I.; Daniel, L.N.; Hassan, A.A. Survey of Cryptosporidium species among ruminants in 
Jos, Plateau state, north-central Nigeria. Journal of Veterinary Advances, 3(2): 49-54, 2013.

Parsons, M.B.; Travis, D.; Lonsdorf, E.V.; Lipende, I.; Roellig, D.M.A.; Kamenya, S.; Gillespie, T.R. Epidemiology and molecular characterization of Cryptosporidium spp. in humans, wild primates, and domesticated animals in the Greater Gombe Ecosystem, Tanzania. PLoS Neglected Tropical Diseases, 9(2): 1-13, 2015.

Paul, S.; Sharma, D.K.; Boral, R.; Mishra, A.K.; Nayakwadi, S.; Banerjee, P.S.; Pawaiya, R.S. Cryptosporidiosis in goats: a review. Journal Advanced Veterinary Animal Science, 2(35): 49-54, 2014.

Pereira, C.R.A.; Ferreira, A.P. Ocorrência e fatores de risco da criptosporidiose em felinos de companhia de idosos. Revista Brasileira de Geriatria e Gerontologia, 15(4): 681-691, 2012.

Quílez, J.; Torres, E.; Chalmers, R.M.; Hadfield, S.J.; Del Cacho, E.; Sánchez Acedo, C. Genotype and subtype characterization of Cryptosporidium in lambs and goat kids in Spain. Applied and Environmental Microbiology, 79(19): 60266031, 2008.

Rieux, A.; Parauda, C.; Porsa, I.; Chartier, C. Molecular characterization of Cryptosporidium spp. in pre-weaned kids in a dairy goat farm in western France. Veterinary Parasitology, Amsterdam, 192(1-3): 268-272, 2013.

Rodrigues, R.D.; Gomes, L.R.; de Souza, R.R.; Barbosa, F.C. Comparação da eficiência das colorações de Ziehl-Neelsen modificado e Safranina modificada na detecção de oocistos de Cryptosporidium spp. (Eucoccidiorida, Cryptosporidiidae) a partir de amostras fecais de bezerros de 0 a 3 meses. Ciência Animal Brasileira, 17(1): 119-125, 2016.

Rossi, G.A.M.; Hoppe, E.G.L.; Martins, A.M.C.V.; Prata, L.F. Zoonoses parasitárias veiculadas por alimentos de origem animal: revisão sobre a situação no Brasil. Arquivos do Instituto Biológico, 81(3): 290-298, 2014.

Smith, H.V.; Caccio, S.M.; Cook, N.; Nichols, R.A.B.; Tait, A. Cryptosporidium and Giardia as foodborne zoonoses. Veterinary Parasitology, 149: 29-40, 2007.

Soltane, R.; Guyot, K.; Dei-Cas, E.; Ayadi, A. Prevalence of Cryptosporidium spp. (Eucoccidiorida: Cryptosporiidae) in seven species of farm animals of Tunisia. Parasite, 14(4): 335-338, 2007.

Teixeira, W.C.; Santos, H.P.; da Silva, J.C.R.; Rizzo, H.; Marvulo, M.F.V.; de Castro, R.S. Perfil zoosanitário dos rebanhos caprinos e ovinos em três mesorregiões do estado do Maranhão, Brasil. Acta Veterinaria Brasilica, 9(1): 34-42, 2015.

Tyzzer, E.E. A Sporozoan found in the peptic glands of the common mouse. Proceedings of the Society for Experimental Biology and Medicine, 5(1): 12-13, 1907.

Tyzzer, E.E. Cryptosporidium parvum (sp. nov.) a coccidium found in the small intestine of the common mouse. Archiv für Protistenkunde, 26: 394-412, 1912.

Tzanidakis, N.; Sotiraki, S.; Claerebout, E.; Ehsan, A.; Voutzourakis, N.; Kostopoulou, D.; Geurden, T. Occurrence and molecular characterization of Giardia duodenalis and Cryptosporidium spp. in sheep and goats reared under dairy husbandry systems in Greece. Parasite, 21(45): 1-7, 2014.

Viel, H.; Rocques, H.; Martin, J.; Chartier, C. Efficacy of nitazoxanide against experimental cryptosporidiosis in goat neonates. Parasitology Research, 102(1): 163-166, 2007.

Wang, L.; Zhang, H.; Zhao, X.; Zhang, L.; Zhang, G.; Guo, M.; Xiao, L. Zoonotic Cryptosporidium species and Enterocytozoon bieneusi genotypes in HIV-positive patients on antiretroviral therapy. Journal of Clinical Microbiology, 51(2): 1-30, 2012. 